\title{
Neurodegenerative disorders and gut-brain interactions
}

\author{
Alpana Singh, ${ }^{1}$ Ted M. Dawson, ${ }^{2,3,4,5,6}$ and Subhash Kulkarni' \\ ${ }^{1}$ Center for Neurogastroenterology, Division of Gastroenterology and Hepatology, Department of Medicine, ${ }^{2}$ Neuroregeneration and Stem Cell Programs, Institute for Cell Engineering, ${ }^{3}$ Department of \\ Neurology, ${ }^{4}$ Solomon H. Snyder Department of Neuroscience, and ${ }^{5}$ Department of Pharmacology and Molecular Sciences, Johns Hopkins University School of Medicine, Baltimore, Maryland, USA. \\ ${ }^{6}$ Adrienne Helis Malvin Medical Research Foundation, New Orleans, Louisiana, USA.
}

\begin{abstract}
Neurodegenerative disorders (NDs) affect essential functions not only in the CNS, but also cause persistent gut dysfunctions, suggesting that they have an impact on both CNS and gut-innervating neurons. Although the CNS biology of NDs continues to be well studied, how gut-innervating neurons, including those that connect the gut to the brain, are affected by or involved in the etiology of these debilitating and progressive disorders has been understudied. Studies in recent years have shown how CNS and gut biology, aided by the gut-brain connecting neurons, modulate each other's functions. These studies underscore the importance of exploring the gut-innervating and gut-brain connecting neurons of the CNS and gut function in health, as well as the etiology and progression of dysfunction in NDs. In this Review, we discuss our current understanding of how the various gut-innervating neurons and gut physiology are involved in the etiology of NDs, including Parkinson's disease, Alzheimer's disease, Huntington's disease, and amyotrophic lateral sclerosis, to cause progressive CNS and persistent gut dysfunction.
\end{abstract}

Neurodegenerative disorders (NDs) are chronic and progressive disorders that disproportionately affect the elderly and have been characterized by selective loss of neurons in the CNS (1). Their prevalence is increasing - partly owing to extensions in lifespan - and by 2030, individuals affected by NDs will account for more than 8 million patients in the United States (2). Various NDs can be characterized and differentiated by their primary clinical features, the anatomical location of the neurodegeneration, the various cell types they affect, and/or the principal molecular abnormality that causes them (3).

Prior to the considerable progress made in recent years that will be discussed here, psychiatrists, neurologists, and gastroenterologists alike supported the idea of the existence of a disease

Conflict of interest: TMD has received personal compensation as a consultant for Sun Pharma Advanced Research Company; is a member of the advisory board of American Gene Technologies International Inc.; and is a consultant for Mitokinin and Inhibikase Therapeutics Inc. TMD is compensated for his roles as a consultant for and interim chief scientific officer of Valted Seq Inc., and his spouse serves on the board of directors and is compensated for her role as interim CEO of Valted Seq Inc. TMD was a paid consultant to and advisory board member of DONG-A ST and has received personal compensation for consulting and serving on the scientific advisory board of Hopstem Inc. TMD owns stock options in American Gene Technologies International Inc., Mitokinin, and Inhibikase Therapeutics Inc. TMD is a founder of Valted LLC and holds an ownership equity interest in the company. TMD is an inventor of technology for Neuraly Inc. that has been optioned from Johns Hopkins University. TMD and his spouse are founders of and hold shares of stock as well as equity in Neuraly Inc., which is now a subsidiary of D\&D Pharmatech. TMD and his spouse hold shares of stock as well as equity in D\&D Pharmatech. TMD and his spouse are founders of and hold equity in Valted Seq Inc.

Copyright: (๖) 2021, American Society for Clinical Investigation.

Reference information: J Clin Invest. 2021;131(13):e143775.

https://doi.org/10.1172/JCl143775. called the "institutional colon." This term described the presence of an amotile and/or elongated and largely distended colon with resulting gut dysfunctions in psychiatric patients who lived in mental health institutions (4). That such a disease existed was often questioned by contemporary physicians, and the confluence of gut and behavioral dysfunction was ascribed to the side effects of medicines, incorrect or inadequate diets, or inattention. To prove that the "institutional colon" is a true disease, Sonnenberg et al. (4) combed through millions of medical records at the US Veterans Affairs to show that patients with presenile dementia, Alzheimer's disease (AD), Parkinson's disease (PD), amyotrophic lateral sclerosis (ALS), or Huntington's disease (HD) had significantly higher odds of also experiencing colonic dysfunction. The authors pointed out that "not all neurons involved in control of intestinal motility are located within the enteric nervous system, but may originate, for instance, in the vagal nuclei at the medulla oblongata or in the sacral segments of the spinal cord" and that "any referral to the loss of neuronal control of colonic motility does not allude to a common pathway, and leaves a multitude of heterogeneous mechanisms possible" (4). This landmark study showing the involvement of both gut and brain dysfunction in NDs paved the way for the studies discussed in this Review.

Here, we review the processes associated with ND etiology and how various gut-innervating and gut-brain connecting neurons are either affected in NDs or are involved in their etiology. We also review the role of microbiota in driving NDs and discuss some open questions in this field. This Review will focus on the classical NDs - PD, AD, HD, and ALS - given that these are not only the major NDs but are also the diseases for which substantial information is available about associated gut dysfunctions. 


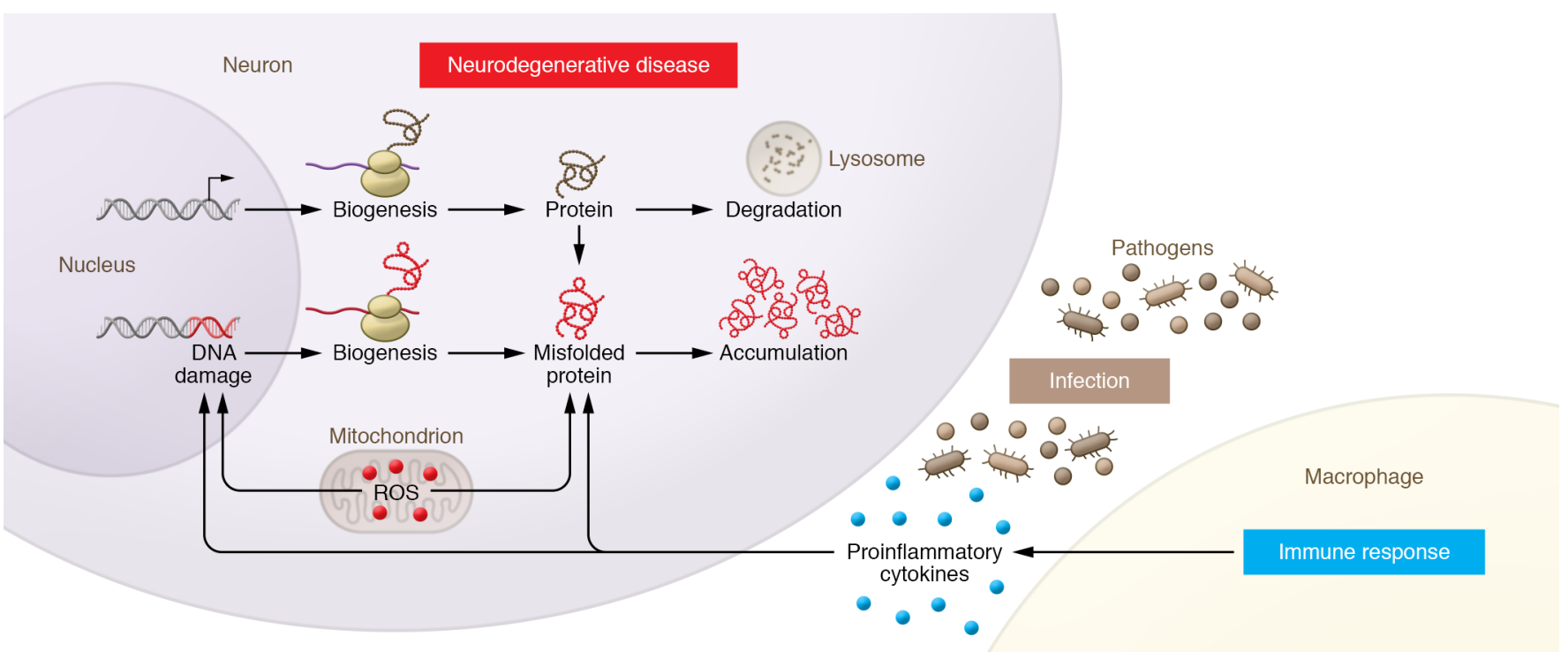

Figure 1. Intra- and extracellular stressors drive NDs. Pathological alterations in normal homeostatic mechanisms of protein genesis, trafficking, and degradation cause the accumulation of misfolded proteins. During the action of normal homeostatic mechanisms, polypeptides are translated outside the nucleus from mRNA transcribed from nuclear DNA and are then folded normally and trafficked to specific locations within the cells. Old proteins are marked for degradation and are trafficked to the lysosome for degradation. In cases of DNA mutations, the translated polypeptides have a higher probability of folding incorrectly, thereby generating degradation-resistant proteins that accumulate to drive pathologies. In addition, intracellular stressors, such as an abundance of ROS generated within cellular mitochondria, or extracellular stressors, such as infections or aberrant proinflammatory cytokine responses to these infections from immune cells, may cause aberrant biogenesis or misfolding of proteins, thus leading to their accumulation. Illustration adapted with permission from Noelle Burgess at the Institute for Cellular Engineering of Johns Hopkins University.

\section{Molecular mechanisms driving NDs}

The molecular mechanisms of NDs can be differentiated as either cell intrinsic or cell extrinsic (Figure 1). The cell-intrinsic mechanisms include proteostatic stress, inherent protein degradation abnormalities, oxidative stress, and heritable mutations.

Proteostatic stress causing protein misfolding. Stress on proteostasis, the dynamic regulation of a dynamic and balanced proteome, may adversely affect the biogenesis, trafficking, folding, and degradation of proteins, resulting in the genesis and accumulation of defective proteins that, in part, drive ND pathologies (5-9).

Inherent abnormalities in protein degradation. Misfolded proteins are proteolytically degraded via ubiquitin-proteasomal degradation, chaperone-mediated autophagy, lysosomal degradation, and macroautophagy pathways. Mutations in the genes encoding proteins in these pathways may cause NDs as a result of inefficient degradation of defective proteins (10-21);

Oxidative stress. ROS, which are constantly produced in aerobic cells as byproducts of normal oxygen metabolism, are rapidly removed by several cellular processes, the dysfunction of which can promote the development of pathological proteins (22-25).

Heritable mutations. Heritable mutations cause the genesis of defective proteins that are either prone to misfolding, form pathological fragmented proteins, or hamper critical pathways that are associated with the development of various $\operatorname{NDs}(1,13,26,27)$.

In contrast, cell-extrinsic mechanisms are associated with infections and/or aberrant immune responses (Figure 1). Primary infections or aberrant immune responses can contribute to ND progression $(28,29)$. Apart from infections, microbial dysbiosis is also known to drive ND pathologies by altering host cell behavior
(30), suggesting that dysregulated cell-extrinsic factors can cause pathological changes in cell-intrinsic pathways.

Age is the most common risk factor for NDs, given that our cumulative exposure to cell-intrinsic and -extrinsic factors increases with age (31). In addition, acute onset of these dysregulated factors may act as a "second hit" in aged individuals, increasing their susceptibility to developing NDs.

\section{Gut-innervating neurons}

The gut is innervated by diverse neuronal populations, including neurons of the enteric nervous system (ENS; residing within the gut wall), spinal nociceptive neurons (residing within the dorsal root ganglia [DRG]), sensory vagal neurons (residing within the nodose ganglia [NG]), extrinsic sympathetic neurons (residing within the sympathetic ganglia [SG]), and efferent neurons of the vagus nerve (residing in the dorsal motor vagal [DMV] nucleus in the brainstem) (Figure 2 and ref. 32). Except for vagal neurons, other gut-innervating neurons are part of the PNS. The ENS, which is the largest subdivision of the PNS, is derived at birth from the embryonic neural crest (33) and is composed of the myenteric and the submucosal plexus, which run parallel through almost the entire length of the gut. The adult ENS remains structurally stable even though it inhabits an organ that subjects it to considerable mechanical, chemical, and microbial stressors. Stability of the adult ENS is possible because almost the entire neuronal population of the ENS is continually renewed every few weeks, driven by a population of nestin ${ }^{+}$enteric neural precursor cells (ENPCs) that continuously and rapidly generate new neurons to offset the neuronal populations lost due to apoptosis (34). 


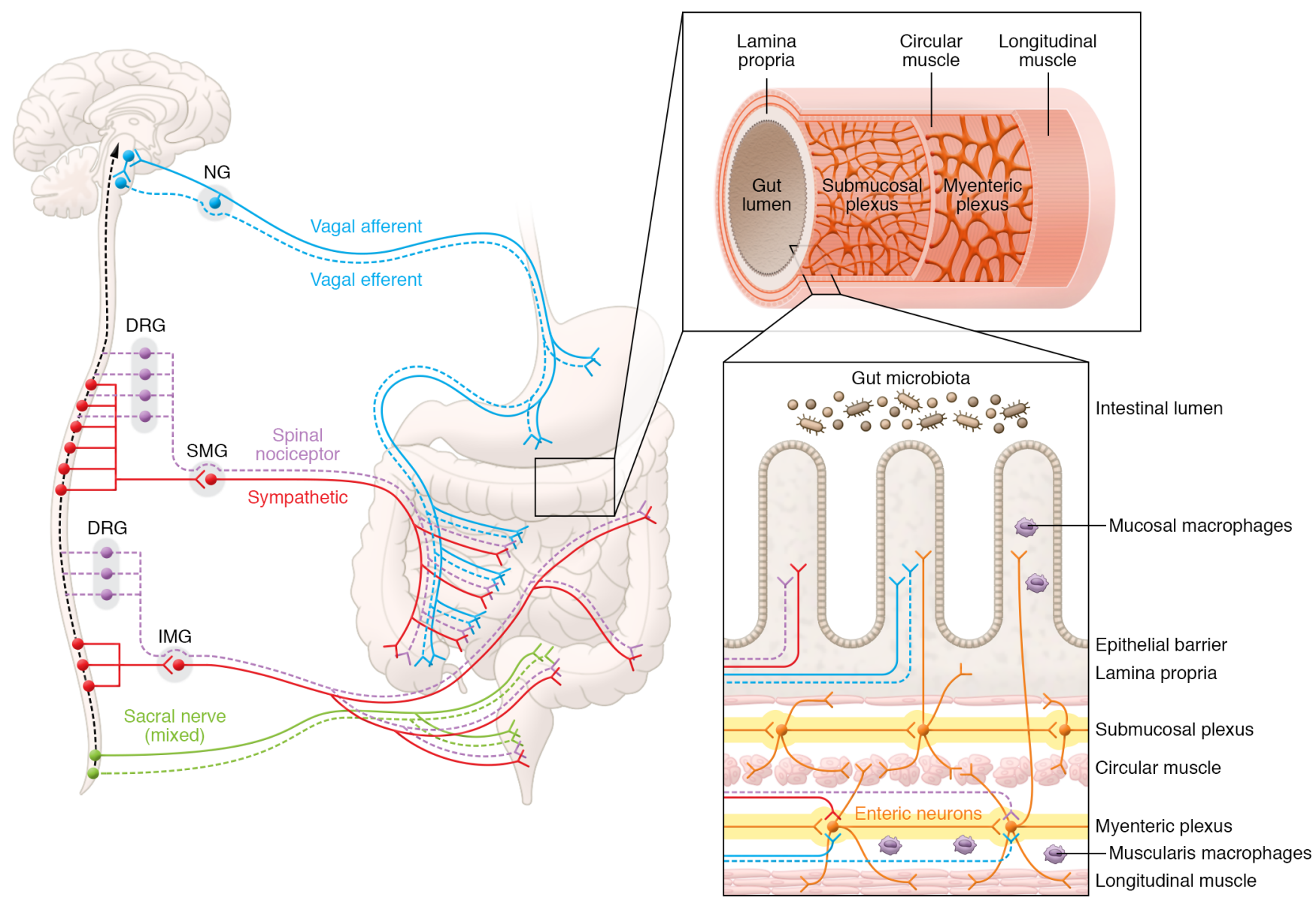

Figure 2. Neuronal connectome between the CNS and the gut. The CNS is connected to the gut, both through its interactions with the ENS and independently through interaction with diverse gastrointestinal cells. The neural pathways that connect the CNS to the gut include the vagus nerve, which consists of the vagal afferent nerve (whose neurons reside in the bilateral NG) and the vagal efferent nerve (whose neurons reside in the brainstem); the spinal nociceptive nerves (whose neurons reside in the DRG, which are sensory neurons that innervate the viscera and the spinal cord neurons); the postsynaptic sympathetic nerves (whose neurons reside in sympathetic superior mesenteric ganglia [SMC] and inferior mesenteric ganglia [IMG]); and the spinal sacral nerve, which directly connects the spinal neurons to the colon. Vagal efferent neurons are known to innervate gastric mucosa, but whether they innervate mucosa in other gut regions is yet unknown. The gut has its own ENS, whose neurons are present in two networks or plexuses. The myenteric plexus is present between the longitudinal muscle layer and the circular muscle layer, whereas the submucosal plexus is present between the CM layer and the lamina propria. The submucosal plexus and myenteric plexus neurons innervate various tissue regions, including the mucosa. The barrier function of the gut is regulated by the epithelial cell layer, which protects the gut wall from luminal contents, including intestinal microbiota. Illustration adapted with permission from Noelle Burgess at the Institute for Cellular Engineering of Johns Hopkins University.

\section{The gut-brain axis}

Evolutionarily, it may be argued that the ENS was the "first brain" (35), given that the primordial neural networks dedicated to regulating intestinal functions evolved earlier than the CNS. Over time, executive functions evolved and diverged to form the CNS, while the gut-centric functions remained in these primordial networks to become the ENS. Despite the divergence, the ENS and CNS remain in constant communication through diverse neural networks known as the gut-brain axis (GBA) (Figure 2). In recent years, progress in our understanding of the GBA has come from studies of the vagus nerve, which carries both afferent (80\%-90\%) and efferent (10\%-20\%) nerve fibers. The vagus nerve innervates various visceral organs, including the gut (36), allowing the CNS to regulate specific gut functions, such as gastric motility (37). At the same time, vagus nerve innervation allows the gut to regulate functions, such as satiety and mood, that were long thought to be under the sole control of the CNS $(38,39)$. The evidence that perturbations in the gut precipitate serious mood disorders through the vagus nerve underscores the importance of this component of the GBA in the maintenance of normal behavior (40).

The gut also receives spinal innervation (Figure 2). Thoracolumbar and lumbosacral DRG neurons provide nociceptive innervation to the gut (41) and also project to the second-order spinal neurons, which in turn project to the brainstem. Unlike the bidirectional signaling of the vagus nerve, DRG-mediated circuitry is unidirectional (41). Recently, Lai et al. presented evidence of local immunomodulatory functions of this circuitry in the gut (42), suggesting control of local functions by these neurons, although we do not yet know whether these also involve higher-order neurons that would constitute a CNS-mediated effect. In addition, the gut also receives indirect spinal innervation through post-ganglionic 


\section{Table 1. NDs and their impact on the CNS and ENS}

\begin{tabular}{|c|c|c|c|c|}
\hline Disorder & CNS neurodegeneration & CNS symptoms & ENS neurodegeneration & Gut symptoms \\
\hline PD & $\begin{array}{l}\text { Loss of dopamine neurons ( } 60,61) \text {. DA ventral } \\
\text { tegmental area neurons and norepinephrine } \\
\text { neurons of the locus coeruleus are also affected. } \\
\text { Neuronal degeneration in PD also affects } \\
\text { pigmented and nonpigmented neurons outside } \\
\text { the midbrain and the brainstem and can involve } \\
\text { multiple neurotransmitter systems (161-165) }\end{array}$ & $\begin{array}{l}\text { Motor dysfunction (166-169), dementia } \\
\text { (170), depression (171, 172), anxiety } \\
(173,174) \text {, sleep disorders (175) }\end{array}$ & $\begin{array}{l}\text { None in human tissues or in } \\
\text { animal models }(94,95)\end{array}$ & $\begin{array}{l}\text { Constipation (47, 77, 176-178), } \\
\text { abdominal pain (50), gut inflammation } \\
(179,180) \text {, microbial dysbiosis }(54,181) \text {, } \\
\text { gastroparesis (182), dysphagia (182- } \\
\text { 184), nausea (182), early satiety (185), } \\
\text { increased intestinal permeability (186) }\end{array}$ \\
\hline$A D$ & $\begin{array}{l}\text { Loss of hippocampal entorhinal cortex, posterior } \\
\text { cingulate, and amygdala and other brain regions } \\
\text { in all stages of } A D(187,188), \text { Loss of cholinergic } \\
\text { neurons of the basal forebrain also occurs } \\
(109,110,189)\end{array}$ & $\begin{array}{l}\text { Motor dysfunction (190-193), dementia } \\
(194,195), \text { depression (196), anxiety } \\
\text { (197-199), sleep disorders (200, 201) }\end{array}$ & None in human samples (113) & $\begin{array}{l}\text { Gut dysmotility, leaky gut, intestinal } \\
\text { inflammation, early satiety, microbial } \\
\text { dysbiosis }(30,58,202-207)\end{array}$ \\
\hline$H D$ & $\begin{array}{c}\text { Loss of striatal GABAergic medium-sized spiny } \\
\text { neurons in early disease, widespread loss in later } \\
\text { disease (124) }\end{array}$ & $\begin{array}{l}\text { Motor dysfunction (208, 209), dementia } \\
(210) \text {, depression }(211) \text {, anxiety }(212) \text {, } \\
\text { sleep disorder }(213,214)\end{array}$ & $\begin{array}{l}\text { Significant neurodegeneration } \\
\text { with marked reduction in the } \\
\text { expression of neuropeptides } \\
\text { in a mouse model (125) }\end{array}$ & $\begin{array}{l}\text { Diarrhea (125), leaky gut (215), } \\
\text { dysphagia (216), weight loss (125), } \\
\text { microbial dysbiosis ( } 53)\end{array}$ \\
\hline ALS & Loss of upper and lower motor neurons (129) & $\begin{array}{l}\text { Loss of voluntary muscle } \\
\text { movement (129) }\end{array}$ & $\begin{array}{l}\text { Selective degeneration of NOS1+ } \\
\text { neurons in mouse models (131) }\end{array}$ & $\begin{array}{l}\text { Delayed gastric and colonic motility, } \\
\text { microbial dysbiosis }(52,217,218)\end{array}$ \\
\hline
\end{tabular}

neurons of the sympathetic ganglia (43), as well as direct spinal innervation through the sacral nerve (44).

\section{Impact of NDs beyond the brain}

Apart from CNS dysfunction, patients with NDs experience a substantial reduction in their quality of life as a result of a significant loss of gut function causing constipation (45-48), abdominal pain (49-52), and microbial dysbiosis (30, 53-59) that can be debilitating and progressive. We classify the CNS and gut dysfunctions in various NDs in Table 1 and will review specific NDs to discuss how extra-CNS neurons are affected by or contribute to ND pathobiology. Although the NDs discussed in this Review have known involvement of the GBA in their pathobiology, they are presented here in descending order of our mechanistic knowledge of this GBA involvement.

\section{Parkinson's disease}

PD is a progressive and debilitating ND caused by selective loss of dopaminergic neurons in the substantia nigra par compacta region of the midbrain that leads to striatal dopamine depletion $(60,61)$. Patients with PD experience CNS dysfunctions including motor and behavioral symptoms, as well as gut dysfunctions (Table 1). An accurate pathologic diagnosis of PD is possible only after a postmortem brain autopsy reveals the presence of inclusion bodies made up of aggregated, misfolded $\alpha$-synuclein protein $(\alpha$-SYN), termed Lewy body pathology (LP) (62-71). However, LP is not restricted to the brain tissue of patients with PD, but is also present in their ENS, suggesting the involvement of ENS neurons $(72,73)$.

The presence of symptoms and LP within and outside of the CNS led to questions of how and where LP forms, and how it affects intra- and extra-CNS neurons. The key to understanding this came in 2003 from Braak et al., who studied LP in postmortem brain tissue from patients with PD and classified the location and abundance of LP in brain tissue by the patients' symptom severity (74). They observed that, although LP was present mostly in the lower brainstem in patients with less severe disease, its presence and abundance moved deeper into the midbrain with increasing severity of disease (74). Braak and colleagues staged PD progression on the basis of the presence of LP in the lower brainstem (stage 1), the pontine tegmentum (stage 2), the midbrain (stage 3), the mesocortex (stage 4), and the neocortex (stages 5 and 6). In addition, the presence of LP in the brainstem and ENS in early stages of the disease led the researchers to hypothesize that LP may originate in the gut, where it spreads to the CNS via anatomically connected neurons (ref. 75 and Figure 3). This could potentially explain not only why patients with PD experience gut dysfunction before they even develop CNS symptoms $(62,65,76-80)$, but also why the surgical severance of the vagus nerve confers an apparently protective effect or reduces the risk of developing PD $(81,82)$.

Following the discovery by Braak et al., initial investigations focused on how gut dysfunction occurs in established models of CNS-directed PD. In the 6-hydroxydopamine (6-OHDA) model, in which neurotoxic 6-OHDA is delivered directly into the CNS, studies revealed a significant reduction in intestinal motility in the gut associated with a loss of neuronal nitric oxide synthase 1 (NOS1), the enzyme that synthesizes the inhibitory neurotransmitter nitric oxide, and an increase in tyrosine hydroxylase (TH), the rate-limiting enzyme in dopamine synthesis $(83,84)$. That a CNS-specific intervention caused significant changes to the ENS provided evidence that gut dysfunction in PD is not due to CNS disease, but rather involves significant ENS alterations. Similarly, Anselmi et al. showed that subthreshold exposure of the gut to toxins not only impaired gut function, but also led to the presence of misfolded $\alpha$-SYN and an associated loss of dopaminergic neurons in the brain (85). These data suggest a bidirectional transmissibility of pathology between the CNS and the gut.

To query the involvement of the GBA as a conduit for gutbrain transfer of pathology, investigators have injected the gut with human brain lysate from patients with $\mathrm{PD}$ and recombinant $\alpha$-SYN (86), exposed the gut to toxins (85), or injected preformed 


\section{A Bottom-up PD progression}

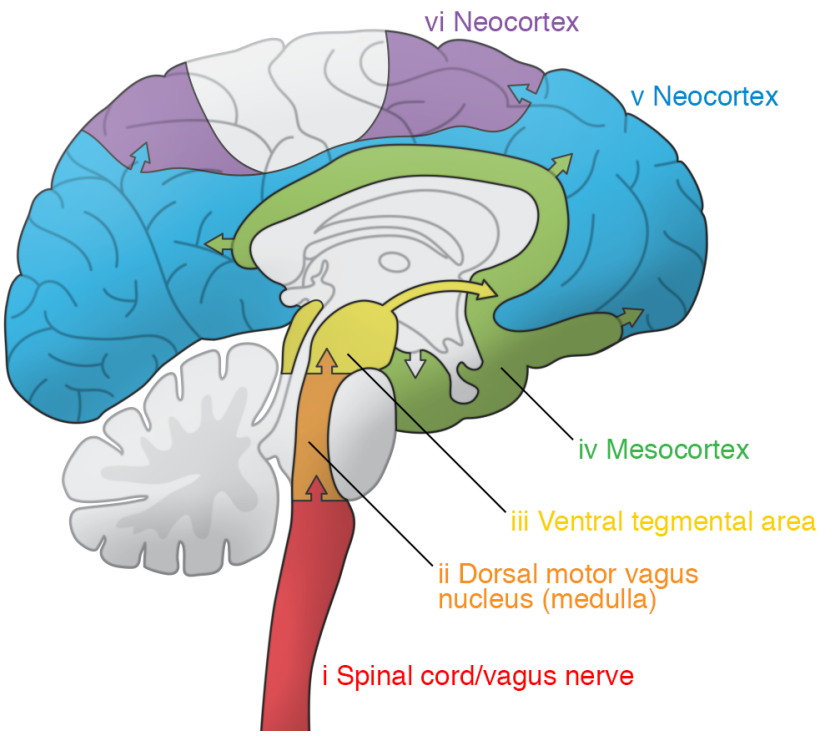

B Top-down PD progression

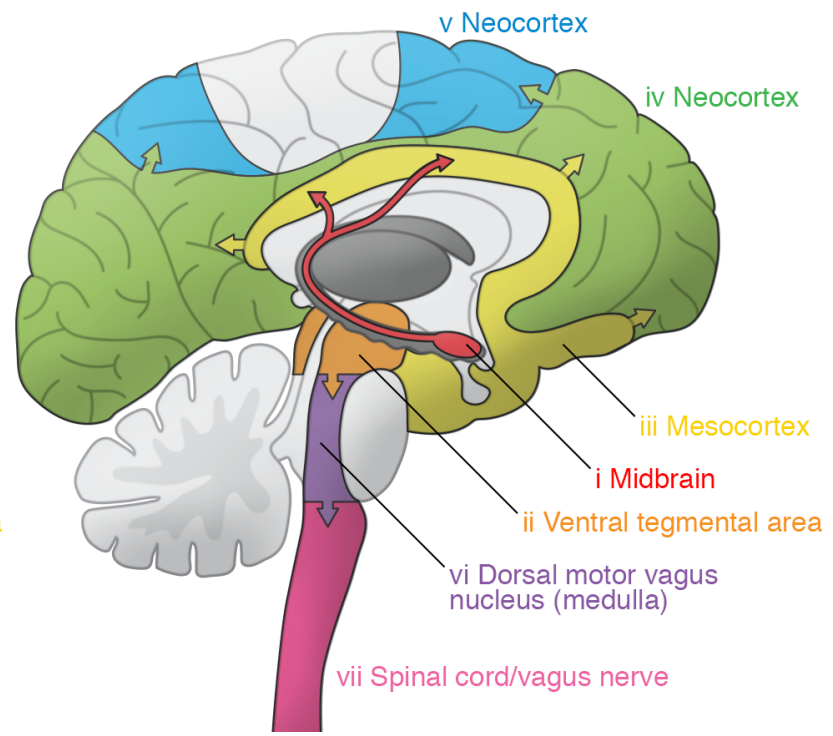

Figure 3. Top-down and bottom-up progression models of PD. The progression of PD in patients is known to follow two different progression patterns. (A) The first follows Braak's stages, where the ascent of the LP occurs from the (i) vagal or sympathetic nerves to the (ii) dorsal motor vagus nucleus in the brainstem, which then progresses to (iii) the ventral tegmental area, (iv) the mesocortex, and (v, vi) the neocortex. (B) On the other hand, the second pattern follows the top-down pathway, which originates near the (i) midbrain and (ii) ventral tegmental area, and then spreads to (iii) the mesocortical and (iv, v) neocortical regions as well as descends the (vi) medulla into the dorsal motor vagus nucleus in the brainstem, from where it progresses down (vii) the vagus nerve and descends into the spinal cord to spread down the spinal and sympathetic pathways. Illustration adapted with permission from Noelle Burgess at the Institute for Cellular Engineering of Johns Hopkins University.

fibrils (PFFs) of pathological $\alpha$-SYN and viral vectors carrying overexpressed mutant SNCA (encoding $\alpha$-SYN) into the gut wall (87) and observed the transference of gut-based pathology to the brain, which could be avoided by vagotomy. In an independent study by Kim et al., we tested whether the transfer of pathology is dependent on the presence of endogenous $\alpha$-SYN and causes hallmark neurodegeneration and associated symptoms. By inoculating the gut of adult WT mice and SNCA-KO mice with PFFs, we showed that PFF-injected WT mice, but not SNCA-KO mice, develop CNS pathology, midbrain neurodegeneration, and motor function loss (88). Previous reports found that healthy fetal neurons develop PD pathology after engraftment into patients with PD $(89,90)$. The Brundin group, among others, showed in vitro that misfolded $\alpha$-SYN enters healthy cells, where these proteins act as a template upon which endogenous $\alpha$-SYN within healthy cells can misfold and aggregate $(91,92)$. Thus, in Kim et al., we showed that this prion-like behavior is central to the gut-to-brain transmission of PD pathology (88). Further, after vagotomy, PFF-injected mice did not develop PD symptoms or CNS neurodegeneration, providing evidence that PFF-driven gut pathology ascends the vagus nerve and into the CNS to cause loss of dopaminergic neurons and the onset of motor and behavioral symptoms. Thus, we provided experimental verification of Braak's hypothesis of an extra-CNS origin of PD pathology and showed that the vagus nerve is an important route by which the pathology is imported into the CNS. Mechanisms involving the uptake of PFFs by the ENS or the GBA and the subcellular locations of templated aggregation remain unknown and may involve the LAG3 receptor-mediated endocytosis mechanisms observed in CNS neurons (93).
Subsequently, Challis et al. (94) used a similar gut inoculation model to show that, although PD-like CNS pathology develops in aged but not younger mice, persistent gut dysfunction occurs without significant and persistent structural changes to the ENS. The occurrence of the CNS, but not ENS, neurodegeneration observed here simulates the lack of ENS neurodegeneration observed upon pathological assessment of postmortem intestinal tissues of patients with PD (95). While preservation of the ENS structure in the presence of PD pathology could be explained by the ENS neurogenic processes discussed above (34), how intestinal dysfunction persists despite a normal ENS structure in patients with PD is yet unknown, but it is plausible that this involves tenacious dysfunction of newborn neurons in response to exacerbated tissue pathology (96).

GWAS have identified approximately 90 PD-associated risk loci including SNCA, LRRK2, PINK1, and PARKIN genes, which represent $16 \%-36 \%$ of the heritable component of the disease (97). However, mutations alone may not be sufficient to cause early-onset PD CNS symptoms. For example, congenital presence of the PD-associated human mutant SNCA transgene in A53T-transgenic mice does not cause early-onset CNS ND, but it does cause extensive ENS dysfunction (98). Why the presence of congenital PD pathology in this model does not result in early-onset CNS pathology, while enteric inoculation with PFFs does, is not known, but it can be hypothesized that the pathology clearance mechanisms in the CNS may be upregulated in younger, but not aged, adults to compensate for the congenital increase in mutant $\alpha$-SYN abundance.

PD does not always originate in the gut, and although some patients follow a gut-to-brain bottom-up progression of PD (called prodromal PD) that follows Braak's stages, others may follow 
a brain-to-gut top-down progression. Prior reports show that a CNS-centric intervention caused the development of PD pathology first in the CNS, and then caused significant gut neurochemical and physiological changes (refs. 99, 100 and Figure 3). Recently, Horsager et al. (101) used multimodal imaging on PD patients with and without a rapid eye movement (REM) sleep behavior disorder (RBD), a marker for prodromal PD, to assess CNS neuronal dysfunction corresponding to Braak's stages. Horsager et al. found that PD patients with RBD followed Braak's stages, suggesting that their disease progressed bottom-up, whereas the disease in patients without RBD progressed top-down, since their pattern did not follow Braak's stages. This not only shows that RBD can be used as a marker to distinguish the two forms of PD, but that imaging-based analyses can be used to track and stratify the progression of disease in living patients.

Patients with PD also experience substantial hyperalgesia (102), suggesting alterations in nociceptive spinal circuits of the GBA. In a rat model of PD, pathological $\alpha$-SYN was found in celiac ganglia of the sympathetic system and in the intermediolateral nucleus of the spinal cord, indicating that PD pathology may spread from the gut to the brain or vice versa through nonvagal circuits, and that these alternate routes of trafficking could be important for the development of significant nonmotor symptoms in PD (103).

Although the present Review suggests that the transmission of PD pathology occurs principally through neural connections, those may not be the only pathways responsible. The possibility of alternate pathways, which may include glymphatic, immunologic, endothelial, and/or cerebrospinal circulation pathways, is especially significant, given the observation of a lack of pathology in some of the "nearest neighbors" to the Lewy pathology-afflicted nuclei in the brainstem and diencephalon regions of patients with advanced PD (104).

\section{Alzheimer's disease}

$\mathrm{AD}$ is the most common ND and is associated with mutations in amyloid precursor protein (APP) and presenilin 1 (PSEN1) genes $(26,105-108)$. AD, which is rarely found without other neurodegenerative copathologies, is generally associated with the presence of amyloid $\beta$ (A $\beta$ ) plaques and neurofibrillary tangles containing hyperphosphorylated TAU protein that cause loss of cholinergic neurons of the basal forebrain as well as of additional CNS neurons $(109,110)$. A $\beta$ plaques are formed by accumulation of the highly fibrillogenic $A \beta$ peptides that result from the abnormal processing of APP by the $\beta$ - and $\gamma$-secretases and an imbalance in the production and clearance pathways (109).

Patients with AD exhibit diverse CNS and ENS dysfunctions (Table 1). Since ENS neurons, the majority of which are cholinergic, also express APP $(111,112)$, it would be reasonable to expect that the ENS would mirror the CNS in losing cholinergic neurons. However, pathological examination of the ENS in patients with AD showed no disease-associated neurodegeneration (113), suggesting that adult neurogenic programs may be responsible for sustaining ENS structure (34). Studies using AD animal models suggest that genetic factors, either alone or in combination with altered intestinal environment, are responsible for the gut dysfunction and an exacerbation of AD pathology in the CNS (114117). The ENS of APP/PS1 double-transgenic mice, which express chimeric mouse/human APP and mutant human PSEN1 genes, shows significant changes in the neurotransmitter expression profiles while preserving the ENS structure (116). Using another AD mouse model, App ${ }^{\mathrm{NL}-\mathrm{G}-\mathrm{F}}$ (in which expression of a triply mutated, humanized APP gene elevates pathogenic $A \beta$ rather than overexpression of the gene), Sohrabi et al. showed that chemically induced intestinal inflammation in animals with these mutations caused enhanced plaque deposition in the CNS (115).

These data lead to the hypothesis that AD pathology may also originate in the gut, which is supported by a report by Lin et al., who assessed the incidence of dementia in patients with truncal vagotomy and concluded that vagotomy reduced the risk of developing dementia (118). Experimental validation of the involvement of the vagus nerve in gut-to-brain trafficking of $\mathrm{AD}$ pathology came from Sun et al., who showed that, upon gut inoculation with $A \beta 1$ 1-42 oligomers, $A \beta$ pathology spread not only within the ENS cholinergic neurons causing persistent dysfunction in specific gut regions, but also ascended up the vagus nerve to the brain to cause cognitive defects (119). How A $\beta$ pathology spreads in the ENS and GBA to cause persistent dysfunction only in specific gut regions remains unknown.

A recent report found evidence of significant neurodegeneration in the spinal cord in patients with AD (120). Dugger et al. found that significant proportions of patients with AD had phosphorylated TAU pathology in their spinal cord when compared with patients without AD (121). Interestingly, this study analyzed various spinal segments and found that the presence of pathology decreased from the cervical to sacral regions. Given that neurofibrillary tangles have not been observed in the peripheral ganglia of patients with AD (72), data from Dugger et al. indicate that the spinal pathology may be of CNS origin, which spreads in the tissue in a top-down manner.

\section{Huntington's disease}

Huntington's disease (HD) is an inherited ND caused by mutation of the Huntingtin (HTT) gene $(122,123)$. HTT mutations involve a CAG trinucleotide repeat, which upon elongation is translated into small, fragmented proteins that accumulate as cellular inclusions to cause ND (27). Neurodegeneration in early HD is highly selective for striatal GABAergic medium-sized spiny neurons that project to the substantia nigra and globus pallidus, whereas the later stages show significant atrophy of a broad range of brain regions, causing profound CNS symptoms (Table 1 and ref. 124).

Although HD also affects various gut functions (Table 1), the most prevalent non-neurological symptom in HD is weight loss (125). Since the gut expresses mutant HTT, and HD patients and a HD mouse model show the presence of HD pathology in the ENS $(126,127)$, it can be postulated that the weight loss is due to intestinal dysfunction. Using an R6/2-transgenic mouse model, which expresses a transgene encoding the $5^{\prime}$ end of the human HTT gene with different lengths of CAG repeat expansions, van der Burg et al. showed that the ENS in HD has significant neurodegeneration, a marked reduction in the expression of neuropeptides, and associated intestinal malabsorption that causes weight loss (125). In addition, patients with HD also experience xerostomia (dry mouth), which can cause dysphagia, and using the same R6/2-transgenic mouse line mentioned above, Wood et al. (128) 
showed that hypothalamic neurodegeneration is responsible for altered drinking behavior and dysphagia, thereby causing weight loss. Thus, in HD, neurodegeneration in both the CNS and ENS causes non-neurological symptoms.

\section{Amyotrophic lateral sclerosis}

ALS is a progressive ND characterized by loss of voluntary muscle movement caused by the death of upper motor neurons (in the motor cortex of the brain) and lower motor neurons (in the brainstem and spinal cord) (129). The pathological hallmarks of ALS are TDP- $43^{+}$cytoplasmic inclusion bodies in motor neurons (130). Patients with ALS also have significant gut dysfunction including delayed gastric and colonic motility (Table 1). Using the ALS mouse model that expresses the mutant TDP-43 (TARDBP) gene, Herdewyn et al. (131) showed selective degeneration of the inhibitory $\mathrm{NOS}^{+}$neurons in the myenteric plexus, which caused intestinal obstruction and sudden death. Preservation of enteric NOS1 ${ }^{+}$ neurons requires a stable receptor tyrosine kinase RET signaling system (132), which is altered pathologically in ALS (133). These findings suggest that a common genetic link may exist between the CNS and ENS dysfunction in ALS, which together may contribute to the progressive nature of the disease. In addition, ALS pathologies can be propagated and trafficked between cells, indicating that extra-CNS pathology may be trafficked to the brain through the GBA (134). However, whether ALS pathologies are trafficked through the GBA is unknown.

\section{Intestinal microbiota, intestinal inflammation, and NDs}

Regardless of whether ENS neurodegeneration occurs, large populations of patients with NDs experience gut dysfunction and microbial dysbiosis (Table 1). While studies found a strong correlation between dysbiosis and the incidence of NDs, whether dysbiosis is the cause or effect of dysfunction in NDs was not known (135, 136). In recent years, through stable colonization of germ-free mice with specific or human donor-derived gut microbiota, investigators have tested whether the gut microbiota independently, or in conjunction with other factors, affect NDs. Sampson et al. showed that gut microbiota are essential for developing PD-associated motor and gut dysfunction in $\alpha$-SYN-overexpressing mice (137). The authors proposed that specific microbiota-derived short chain fatty acids (SCFAs) exacerbate $\alpha$-SYN-driven CNS microglial activation to cause motor dysfunction.

The mechanism by which short-chain fatty acids (SCFAs) affect CNS NDs remains unclear. It can be hypothesized that altered vagal activity in response to intestinal SCFAs may alter CNS microglial activation (137-139). Alternatively, it is also plausible that, since SCFAs drive microglial maturation (140), only mature microglia (in microbiota-colonized mice) and not immature microglia (in SCFA-free, germ-free mice) are able to effect neuroinflammation and neurodegeneration when exposed to ND risk factors. This hypothesis is lent further credence by a recent study by Colombo et al., who similarly found that in the APP/ PS1-transgenic AD mouse model, the presence of gut microbiota promotes the development of cerebral $A \beta$ plaques (117). By a series of experiments, the investigators found that microbiota-derived SCFAs promote microglial maturation, activation, and a microglia- specific increase in ApoE production that is thought to increase plaque loads by aiding increased deposition and reduced clearance (117). These data suggest that SCFAs may play a key role in the development of several NDs by regulating CNS neuroinflammation through the non-neuronal mechanisms discussed earlier. Although it may be tempting to implicate SCFAs as the sole driver of disease, other reports that show diametrically opposite effects of SCFAs in the etiology and amelioration of NDs $(141,142)$ suggest that other microbiota-derived factors in conjunction with SCFAs may play a role in disease etiology.

Such factors, which include specific bacterial pathogenic proteins, follow the bottom-up or gut-first pathway. Evidence for this comes from a few recent studies, the foremost of which was from Sampson et al. (143), who showed that specific bacteria abundantly express cell-surface amyloid fibers called CURLI proteins, which, in conjunction with $\alpha$-SYN overexpression, accelerate the development of PD pathology and motor and gut dysfunction. They showed that treatment with a gut-restricted amyloid inhibitor prevents CURLI-mediated progression of disease in this model. It can be hypothesized that the presence of CURLI in the gut drives the accelerated development of enteric $\alpha$-SYN pathology, which can then traffic up the vagus nerve to cause CNS dysfunction. Thus, it is plausible that the presence of such pathogenic signals from a dysbiotic microbiota, when combined with aberrant SCFA expression, may increase the risk of developing NDs in the CNS through neuronal (GBA) and non-neuronal (microglia) mechanisms.

While these studies give us clues about how CNS pathology may develop as a result of dysbiotic microbiota or gut infections, the mechanisms by which chronic gut dysfunction occurs remain unclear. A recent study from our group offers insights into how microbial dysbiosis may cause chronic intestinal disorders. In continuation of an earlier study, in which we observed that continual adult neurogenesis is required to maintain ENS structure and gut function (34), Yarandi et al. (144) tested whether microbial dysbiosis negatively affects the neurogenic homeostatic mechanism. Using an antibiotic-mediated model of dysbiosis, Yarandi et al. showed that loss of gram-positive bacteria drives a reduction in TLR2 signaling on ENPCs, which significantly reduces their neurogenic behavior, causing a loss of enteric neurons and normal gut motility. Although the antibiotic treatment, dysbiosis, and resulting gut dysfunction were transient and reversible in this study, it can be argued that long-term antibiotic treatments cause persistent dysbiosis or selection of pathogenic bacteria, leading to permanent changes in enteric neurogenesis causing irreversible gut dysfunction in patients with ND. Support for this hypothesis comes from epidemiological findings that exposure to broad-spectrum antibiotics elevates the risk of developing PD (145).

In addition, ENS homeostasis is supported by intestinal immune cells called muscularis macrophages (MMs) that continually remove dying neurons and neuronal debris (34). MMs are recruited by ENS neurons (32), and it can be hypothesized that any dysbiosis-driven alterations in ENS structure may hamper MM recruitment, resulting in the accumulation of neuronal debris. Since ENS neurons express $\alpha$-SYN (146), accumulation of neuronal debris may drive nucleation and aggregation of pathological $\alpha$-SYN. Bacterial infections may also affect $\alpha$-SYN aggregation independently of their direct action on the ENS, as they 
may cause gut inflammation (147) that results in significant alterations in MM behavior (148). Inflammation drives MMs away from their housekeeping tasks (149), causing debris accumulation and the genesis of PD pathology. Indeed, Kishimoto et al. showed that chemically induced gut inflammation in the human A53T $\alpha$-SYN mouse model induces altered activation of MM and microglia in the gut and brain, respectively, to cause accelerated CNS neurodegeneration (150). A powerful "second-hit," which would overwhelm inherent fail-safe mechanisms, is often needed to exacerbate PD pathology (151). Such second hits may include infections or dysbiosis and inflammation (152-155), which may destabilize the ENS or GBA to cause ND.

In a subset of patients, microbial dysbiosis may not be the cause of the original pathology, but an effect, and would follow the top-down or brain-first pathway. Gut mucosal immune cells are in a constant cross-talk with gut-innervating vagal, sympathetic, and spinal neurons (32), whose activity is altered in NDs (156). Altered GBA activity in brain-first NDs may drive profound changes in intestinal immunity and barrier functions, promoting both gut dysfunction and changes to the microbiota. While 6-OHDA-induced models of brain-first PD show significant shifts in proportions of specific bacteria (157), whether such dysbiosis is the cause or effect of gut dysfunction remains unknown. It can be argued that the resulting microbial dysbiosis in brainfirst NDs may help perpetuate intestinal dysfunction and create a reservoir of de novo pathology in the gut that can again be trafficked to the CNS.

Thus, evidence strongly shows that abnormal microbiota constitute a risk factor for developing NDs and associated gut dysfunction, through both neuron-dependent and independent mechanisms. Following that logic, it can be hypothesized that reverting dysbiosis may arrest or revert CNS disease in the same manner that the microbiota help normalize ENS structure and function (144). This was recently tested using AD mouse models, in which Sun et al. and Kim et al. independently showed that transplantation with normal microbiota reverted $\mathrm{AD}$-associated dysbiosis, macrophage dysfunction, and SCFA levels in the gut and reduced the deposition of $A \beta$ and TAU in the brain to improve cognitive deficits $(158,159)$.

\section{Open questions and future directions}

In the course of this Review, we have identified gaps in our knowledge regarding the etiology and impact of NDs. Some of these gaps are summarized below.

Differences between gut-first and brain-first etiology of $P D$. While there is evidence that shows a dichotomy between top-down and bottom-up PD etiologies, whether the same GBA circuits are involved in the transmission of pathologies in these two PD groups is unclear. It is unknown why there is a significant difference in representation of these two etiologies, as was shown in Finnish patients, the majority of whom had bottom-up progression patterns, while a minority had top-down progression patterns (160). It can be hypothesized that, since the vagus nerve consists mostly of afferent fibers (which take signals from the gut to the brain) as opposed to efferent fibers (which transmit signals from the brain to the gut), the proportions of gut-to-brain transmission of pathologies are higher, suggesting that the GBA circuits involved in the two etiologies are different. Since vagal NG neurons would then drive bottom-up transmission, this hypothesis could be tested by injecting labeled PFFs into the brain or the gut and then observing whether PFFs appear in NG neurons prior to reaching the gut or the brain, respectively. These experiments, along with subsequent experiments utilizing transgenic animals to manipulate afferent and efferent vagal fibers, can provide insight into the differences in top-down versus bottom-up PD etiologies.

ENS and GBA circuits involved in trafficking diverse ND pathologies. In addition to our incomplete understanding of their specific role in PD, we currently lack clarity on whether ENS neurons also help spread ND pathology, and whether the transmission of diverse ND pathologies occurs through the same ENS and GBA circuits. Although both $\mathrm{AD}$ and $\mathrm{PD}$ pathologies utilize the vagus nerve to gain access to the CNS, it is not known whether the same vagal circuits and neuronal subtypes are involved in the trafficking of diverse pathologies. Whether the same neuronal cells are involved in the propagation and transmission of pathology, or whether these occur separately in different cells is unknown. Further, it is unknown whether the ENS plays any role in the transmission and maintenance of a reservoir of pathology. A cross-disease assessment of GBA and ENS circuits involved in the retention and transmission of these pathologies should be performed to understand their role.

The nature of cellular and molecular pathologies underlying gut dysfunction. The persistence of gut dysfunction while the ENS structure remains intact in some NDs suggests that homeostatic mechanisms still cause profound neurochemical and molecular changes in ENS neurons. While continual neurogenesis might repair ENS neuronal loss that is elevated in the gut of individuals with NDs, this should be tested by performing BrdU-labeling experiments and apoptosis assays to calculate the rate of neuronal turnover. In addition, alterations to ENS and GBA neurons at the neurochemical and molecular levels should be studied using immunohistochemical, physiological, and newer single-cell transcriptomic techniques.

Mechanistic role of microbiota in causing or reverting NDs. Although the microbiota play a notable role in NDs, their exact nature and mechanistic contribution are unknown. Although the presence of gut bacteria in mutant mice aid in the progression of some NDs, specific bacteria or microbial communities may play a beneficial role in arresting or even reverting other NDs. A better understanding of their role will require mechanistic insights into how microbiota regulate the ENS and the associated MMs maintain gut function, as well as how they stimulate the GBA in the context of ND mouse models.

Gut- or GBA-centric therapies that can arrest or revert CNS neurodegeneration. Clinically, there are no available disease-modifying therapies to normalize gut function in NDs. It can be postulated that if the ENS or gut becomes a reservoir for pathology, then normalizing this tissue/organ may have a profound effect on normalizing CNS functions in ND. Hence, better therapies for the normalization of ENS structure and function are needed. In recent years, devices for modulating the GBA have come to the fore. The ability of these devices to normalize gut, GBA, and brain function to slow disease progression and lead to "curative" interventions for NDs should be investigated. 


\section{Conclusions}

In this Review, we discussed that, while diverse NDs have similar symptoms, they differ in their etiology and underlying molecular and cellular pathobiology. Although we have come a long way in understanding the factors that underlie the "institutional colon," crafting potential disease-modifying cures that can benefit the behavioral, motor, and gastrointestinal dysfunction in patients with NDs will require a better understanding of how normal and altered gut biology affect the neurons and other cells that reside within and outside of the gut. This is important, since pathology in any one part of this gut-brain continuum negatively affects the specialized and common functions regulated by both the CNS and the ENS.

\section{Acknowledgments}

This work was supported by grants from the National Institute of Neurological Disorders and Stroke (NINDS), NIH (NS38377) and the JPB Foundation. The authors acknowledge joint participa- tion by the Adrienne Helis Malvin Medical Research Foundation through its direct engagement in the continuous active conduct of medical research in conjunction with the Johns Hopkins Hospital, the Johns Hopkins University School of Medicine, and the Foundation's Parkinson's Disease Program (M-2014, to TMD). The authors also acknowledge support from the Ludwig Foundation and the National Institute on Aging (NIA), NIH (1R01AG06676801A1, to SK). TMD is the Lenard and Madlyn Abramson Professor in Neurodegenerative Diseases.

Address correspondence to: Subhash Kulkarni, Center for Neurogastroenterology, Johns Hopkins University School of Medicine, 720 Rutland Avenue, ROSS 935, Baltimore, MD 21205, USA. Email: skulkar9@jh.edu. Or to: Ted M. Dawson, Institute for Cell Engineering, Johns Hopkins University School of Medicine, 733 North Broadway, MRB 731, Baltimore, MD 21205, USA. Email: tdawson@jhmi.edu.
1. Martin JB. Molecular basis of the neurodegenerative disorders. $N$ Engl J Med. 1999;340(25):1970-1980.

2. Dorsey ER, et al. The coming crisis: obtaining care for the growing burden of neurodegenerative conditions. Neurology. 2013;80(21):1989-1996.

3. Dugger BN, Dickson DW. Pathology of neurodegenerative diseases. Cold Spring Harb Perspect Biol. 2017;9(7):a028035.

4. Sonnenberg A, et al. The "institutional colon": a frequent colonic dysmotility in psychiatric and neurologic disease. Am J Gastroenterol. 1994;89(1):62-66.

5 . Kurtishi A, et al. Cellular proteostasis in neurodegeneration. Mol Neurobiol. 2019;56(5):3676-3689.

6 . Yerbury JJ, et al. Walking the tightrope: proteostasis and neurodegenerative disease. JNeurochem. 2016;137(4):489-505.

7. Gandhi J, et al. Protein misfolding and aggregation in neurodegenerative diseases: a review of pathogeneses, novel detection strategies, and potential therapeutics. Rev Neurosci. 2019;30(4):339-358.

8. Soto C, Pritzkow S. Protein misfolding, aggregation, and conformational strains in neurodegenerative diseases. Nat Neurosci. 2018;21(10):1332-1340.

9. Sweeney P, et al. Protein misfolding in neurodegenerative diseases: implications and strategies. Transl Neurodegener. 2017;6:6.

10. Rao G, et al. Ubiquitin-proteasome system in neurodegenerative disorders. J Drug Metab Toxicol. 2015;6(4):187.

11. McKinnon C, Tabrizi SJ. The ubiquitin-proteasome system in neurodegeneration. Antioxid Redox Signal. 2014;21(17):2302-2321.

12. Matsuda N, Tanaka K. Does impairment of the ubiquitin-proteasome system or the autophagylysosome pathway predispose individuals to neurodegenerative disorders such as Parkinson's disease? J Alzheimers Dis. 2010;19(1):1-9.

13. Dawson TM. Parkin and defective ubiquitination in Parkinson's disease. J Neural Transm Suppl. 2006;(70):209-213.

14. Zhang Y, et al. The role of ubiquitin proteasomal system and autophagy-lysosome pathway in Alz- heimer's disease. Rev Neurosci. 2017;28(8):861-868.

15. Monaco A, Fraldi A. Protein aggregation and dysfunction of autophagy-lysosomal pathway: a vicious cycle in lysosomal storage diseases. Front Mol Neurosci. 2020;13:37.

16. Darios F, Stevanin G. Impairment of lysosome function and autophagy in rare neurodegenerative diseases. J Mol Biol. 2020;432(8):2714-2734.

17. Malik BR, et al. Autophagic and endo-lysosomal dysfunction in neurodegenerative disease. $\mathrm{Mol}$ Brain. 2019;12(1):100.

18. Koh JY, et al. Lysosomal dysfunction in proteinopathic neurodegenerative disorders: possible therapeutic roles of cAMP and zinc. Mol Brain. 2019;12(1):18.

19. Wang C, et al. Endo-lysosomal dysfunction: a converging mechanism in neurodegenerative diseases. Curr Opin Neurobiol. 2018;48:52-58.

20. Usenovic M, Krainc D. Lysosomal dysfunction in neurodegeneration: the role of ATP13A2/PARK9. Autophagy. 2012;8(6):987-988.

21. Zhang L, et al. The lysosome and neurodegenerative diseases. Acta Biochim Biophys Sin (Shanghai). 2009;41(6):437-445.

22. Kim GH, et al. The role of oxidative stress in neurodegenerative diseases. Exp Neurobiol. 2015;24(4):325-340.

23. Niedzielska E, et al. Oxidative stress in neurodegenerative diseases. Mol Neurobiol. 2016;53(6):4094-4125.

24. Chen $X$, et al. Oxidative stress in neurodegenerative diseases. Neural Regen Res. 2012;7(5):376-385.

25. Uttara B, et al. Oxidative stress and neurodegenerative diseases: a review of upstream and downstream antioxidant therapeutic options. Curr Neuropharmacol. 2009;7(1):65-74.

26. Murrell J, et al. A mutation in the amyloid precursor protein associated with hereditary Alzheimer's disease. Science. 1991;254(5028):97-99.

27. Saffert $P$, et al. An expanded CAG repeat in huntingtin causes +1 frameshifting. J Biol Chem. 2016;291(35):18505-18513.

28. Dehhaghi M, et al. Microorganisms' footprint in neurodegenerative diseases. Front Cell Neurosci. 2018;12:466.

29. De Chiara G, et al. Infectious agents and neurode- generation. Mol Neurobiol. 2012;46(3):614-638.

30. Sochocka M, et al. The gut microbiome alterations and inflammation-driven pathogenesis of alzheimer's disease-a critical review. Mol Neurobiol. 2019;56(3):1841-1851.

31. Hou Y, et al. Ageing as a risk factor for neurodegenerative disease. Nat Rev Neurol. 2019;15(10):565-581.

32. Kulkarni S, et al. Neuro-innate immune interactions in gut mucosal immunity. Curr Opin Immunol. 2020;68:64-71.

33. Kulkarni S, et al. Advances in enteric neurobiology: the "brain" in the gut in health and disease. J Neurosci. 2018;38(44):9346-9354.

34. Kulkarni S, et al. Adult enteric nervous system in health is maintained by a dynamic balance between neuronal apoptosis and neurogenesis. Proc Natl Acad Sci U S A. 2017;114(18):E3709-E3718.

35. Furness JB, Stebbing MJ. The first brain: Species comparisons and evolutionary implications for the enteric and central nervous systems. Neurogastroenterol Motil. 2018;30(2):e13234.

36. Furness JB. The enteric nervous system and neurogastroenterology. Nat Rev Gastroenterol Hepatol. 2012;9(5):286-294.

37. Travagli RA, Anselmi L. Vagal neurocircuitry and its influence on gastric motility. Nat Rev Gastroenterol Hepatol. 2016;13(7):389-401.

38. Sackeim HA, et al. The long and winding road of vagus nerve stimulation: challenges in developing an intervention for difficult-to-treat mood disorders. Neuropsychiatr Dis Treat. 2020;16:3081-3093.

39. Browning $\mathrm{KN}$, et al. The vagus nerve in appetite regulation, mood, and intestinal inflammation. Gastroenterology. 2017;152(4):730-744.

40. Liu L, et al. Transient gastric irritation in the neonatal rats leads to changes in hypothalamic CRF expression, depression- and anxiety-like behavior as adults. PLoS One. 2011;6(5):e19498.

41. Brookes S, et al. Extrinsic sensory innervation of the gut: structure and function. Adv Exp Med Biol. 2016;891:63-69.

42. Lai NY, et al. Gut-innervating nociceptor neurons regulate peyer's patch microfold cells and SFB levels to mediate salmonella host defense. Cell. 
2020;180(1):33-49.

43. Muller PA, et al. Microbiota modulate sympathetic neurons via a gut-brain circuit. Nature. 2020;583(7816):441-446.

44. Kamm MA, et al. Sacral nerve stimulation for intractable constipation. Gut. 2010;59(3):333-340.

45. Preziosi G, et al. Neurogenic bowel dysfunction in patients with multiple sclerosis: prevalence, impact, and management strategies. Degener Neurol Neuromuscul Dis. 2018;8:79-90.

46. Ofei SY, et al. Constipation burden in children with autism spectrum disorder: emergency department and healthcare use. J Pediatr. 2018;202:12-13.

47. Sauerbier A, Ray Chaudhuri K. Non-motor symptoms: the core of multi-morbid Parkinson's disease. Br J Hosp Med (Lond). 2014;75(1):18-24.

48. Fu A. Neostigmine: an alternative treatment for constipation. Dynamics. 2005;16(1):13-15.

49. Penzol MJ, et al. Functional gastrointestinal disease in autism spectrum disorder: a retrospective descriptive study in a clinical sample. Front Psychiatry. 2019;10:179.

50. Heetun ZS, Quigley EM. Gastroparesis and Parkinson's disease: a systematic review. Parkinsonism Relat Disord. 2012;18(5):433-440.

51. Davies GA, et al. Prion diseases and the gastrointestinal tract. Can J Gastroenterol. 2006;20(1):18-24.

52. Toepfer M, et al. Gastrointestinal dysfunction in amyotrophic lateral sclerosis. Amyotroph Lateral Scler Other Motor Neuron Disord. 1999;1(1):15-19.

53. Wasser CI, et al. Gut dysbiosis in Huntington's disease: associations among gut microbiota, cognitive performance and clinical outcomes. Brain Commun. 2020;2(2):fcaa110.

54. Wallen ZD, et al. Characterizing dysbiosis of gut microbiome in PD: evidence for overabundance of opportunistic pathogens. NPJ Parkinsons Dis. 2020;6:11.

55. Gotkine M, et al. Amyotrophic lateral sclerosis and intestinal microbiota-toward establishing cause and effect. Gut Microbes. 2020;11(6):1833-1841.

56. Schepici G, et al. The gut microbiota in multiple sclerosis: an overview of clinical trials. Cell Transplant. 2019;28(12):1507-1527.

57. Fattorusso A, et al. Autism spectrum disorders and the gut microbiota. Nutrients. 2019;11(3):E521.

58. Wu SC, et al. Intestinal microbial dysbiosis aggravates the progression of Alzheimer's disease in Drosophila. Nat Commun. 2017;8(1):24

59. Donaldson DS, Mabbott NA. The influence of the commensal and pathogenic gut microbiota on prion disease pathogenesis. J Gen Virol. 2016;97(8):1725-1738.

60. Surmeier DJ, et al. What causes the death of dopaminergic neurons in Parkinson's disease? Prog Brain Res. 2010;183:59-77.

61. DauerW, Przedborski S. Parkinson's disease: mechanisms and models. Neuron. 2003;39(6):889-909.

62. Przedborski S. The two-century journey of Parkinson disease research. Nat Rev Neurosci. 2017;18(4):251-259.

63. Reich SG. Diagnosing Parkinson's disease. In: Reich SG, Factor SA, eds. Therapy of Movement Disorders: A Case-Based Approach. Springer;
2019:3-6

64. de Lau LML, Breteler MMB. Epidemiology of Parkinson's disease. Lancet Neurol. 2006;5(6):525-535.

65. Braak $\mathrm{H}$, et al. Staging of brain pathology related to sporadic Parkinson's disease. Neurobiol Aging. 2003;24(2):197-211.

66. Li S, Le W. Milestones of Parkinson's disease research: 200 years of history and beyond. $\mathrm{Neu}$ rosci Bull. 2017;33(5):598-602.

67. Goedert M, et al. 100 years of Lewy pathology. Nat Rev Neurol. 2013;9(1):13-24.

68. Stefanis L. $\alpha$-Synuclein in Parkinson's disease. Cold Spring Harb Perspect Med 2012;2(2):a009399.

69. Vargas JY, et al. The prion-like spreading of $\alpha$-synuclein: From in vitro to in vivo models of Parkinson's disease. Ageing Res Rev. 2019;50:89-101.

70. Shahmoradian SH, et al. Lewy pathology in Parkinson's disease consists of crowded organelles and lipid membranes. Nat Neurosci. 2019;22(7):1099-1109.

71. Kalia LV, Kalia SK. $\alpha$-Synuclein and Lewy pathology in Parkinson's disease. Curr Opin Neurol. 2015;28(4):375-381.

72. Wakabayashi K, et al. Parkinson's disease: the presence of Lewy bodies in Auerbach's and Meissner's plexuses. Acta Neuropathol. 1988;76(3):217-221.

73. Kupsky WJ, et al. Parkinson's disease and megacolon: concentric hyaline inclusions (Lewy bodies) in enteric ganglion cells. Neurology. 1987;37(7):1253-1255.

74. Braak H, et al. Staging of brain pathology related to sporadic Parkinson's disease. Neurobiol Aging. 2003;24(2):197-211.

75. Braak H, et al. Gastric alpha-synuclein immunoreactive inclusions in Meissner's and Auerbach's plexuses in cases staged for Parkinson's disease-related brain pathology. Neurosci Lett. 2006;396(1):67-72.

76. Stirpe P, et al. Constipation: an emerging risk factor for Parkinson's disease? Eur J Neurol. 2016;23(11):1606-1613

77. Abbott RD, et al. Frequency of bowel movements and the future risk of Parkinson's disease. Neurol ogy. 2001;57(3):456-462.

78. Savica R, et al. Medical records documentation of constipation preceding Parkinson disease: a case-control study. Neurology. 2009;73(21):1752-1758.

79. Adams-Carr KL, et al. Constipation preceding Parkinson's disease: a systematic review and meta-analysis. J Neurol Neurosurg Psychiatry. 2015;87(7):710-716.

80. Fu P, et al. Association of intestinal disorders with Parkinson's disease and Alzheimer's disease: a systematic review and meta-analysis. ACS Chemical Neuroscience. 2020;11(3):395-405.

81. Svensson E, et al. Vagotomy and subsequent risk of Parkinson's disease. Ann Neurol. 2015;78(4):522-529.

82. Liu B, et al. Vagotomy and Parkinson disease: a Swedish register-based matched-cohort study. Neurology. 2017;88(21):1996-2002.

83. Zhang $\mathrm{X}$, et al. Alteration of enteric monoamines with monoamine receptors and colonic dysmotility in 6-hydroxydopamine-induced Parkinson's disease rats. Transl Res. 2015;166(2):152-162.

84. Zhu HC, et al. Gastrointestinal dysfunction in a Parkinson's disease rat model and the changes of dopaminergic, nitric oxidergic, and cholinergic neurotransmitters in myenteric plexus. JMol Neurosci. 2012;47(1):15-25.

85. Anselmi L, et al. Ingestion of subthreshold doses of environmental toxins induces ascending Parkinsonism in the rat. NPJ Parkinsons Dis. 2018;4:30.

86. Holmqvist S, et al. Direct evidence of Parkinson pathology spread from the gastrointestinal tract to the brain in rats. Acta Neuropathol. 2014;128(6):805-820.

87. Manfredsson FP, et al. Induction of alpha-synuclein pathology in the enteric nervous system of the rat and non-human primate results in gastrointestinal dysmotility and transient CNS pathology. Neurobiol Dis. 2018;112:106-118.

88. Kim S, et al. Transneuronal propagation of pathologic $\alpha$-synuclein from the gut to the brain models Parkinson's disease. Neuron. 2019;103(4):627-641.

89. Li JY, et al. Lewy bodies in grafted neurons in subjects with Parkinson's disease suggest host-to-graft disease propagation. Nat Med. 2008;14(5):501-503.

90. Kordower JH, et al. Lewy body-like pathology in long-term embryonic nigral transplants in Parkinson's disease. Nat Med. 2008;14(5):504-506.

91. Angot E, Brundin P. Dissecting the potential molecular mechanisms underlying alpha-synuclein cell-to-cell transfer in Parkinson's disease. Parkinsonism Relat Disord. 2009;15(Suppl 3):S143-S147.

92. Angot E, et al. Are synucleinopathies prion-like disorders? Lancet Neurol. 2010;9(11):1128-1138.

93. Mao X, et al. Pathological $\alpha$-synuclein transmission initiated by binding lymphocyte-activation gene 3. Science. 2016;353(6307):aah3374.

94. Challis C, et al. Gut-seeded $\alpha$-synuclein fibrils promote gut dysfunction and brain pathology specifically in aged mice. Nat Neurosci. 2020;23(3):327-336.

95. Annerino DM, et al. Parkinson's disease is not associated with gastrointestinal myenteric ganglion neuron loss. Acta Neuropathol. 2012;124(5):665-680.

96. Baumuratov AS, et al. Enteric neurons from Parkinson's disease patients display ex vivo aberrations in mitochondrial structure. Sci Rep. 2016;6:33117.

97. Bandres-Ciga S, et al. Genetics of Parkinson's disease: an introspection of its journey towards precision medicine. Neurobiol Dis. 2020;137:104782.

98. Kuo YM, et al. Extensive enteric nervous system abnormalities in mice transgenic for artificial chromosomes containing Parkinson diseaseassociated alpha-synuclein gene mutations precede central nervous system changes. Hum Mol Genet. 2010;19(9):1633-1650.

99. Colucci M, et al. Intestinal dysmotility and enteric neurochemical changes in a Parkinson's disease rat model. Auton Neurosci. 2012;169(2):77-86.

100. O'Donovan SM, et al. Nigral overexpression of $\alpha$-synuclein in a rat Parkinson's disease model indicates alterations in the enteric nervous system and the gut microbiome. Neurogastroenterol Motil. 2020;32(1):e13726. 
101. Horsager J, et al. Brain-first versus body-first Parkinson's disease: a multimodal imaging casecontrol study. Brain. 2020;143(10):3077-3088.

102. Buhmann C, et al. Management of pain in Parkinson's disease. J Parkinsons Dis. 2020;10(s1):S37-S48.

103. Van Den Berge N, et al. Evidence for bidirectional and trans-synaptic parasympathetic and sympathetic propagation of alpha-synuclein in rats. Acta Neuropathol. 2019;138(4):535-550.

104. Surmeier DJ, et al. Selective neuronal vulnerability in Parkinson disease. Nat Rev Neurosci. 2017;18(2):101-113.

105. Guerreiro RJ, et al. Genetic screening of Alzheimer's disease genes in Iberian and African samples yields novel mutations in presenilins and APP. Neurobiol Aging. 2010;31(5):725-731.

106. Nelson O, et al. Familial Alzheimer disease-linked mutations specifically disrupt $\mathrm{Ca} 2+$ leak function of presenilin 1.J Clin Invest. 2007;117(5):1230-1239.

107. Hutton M, Hardy J. The presenilins and Alzheimer's disease. Hum Mol Genet. 1997;6(10):1639-1646.

108. Goate A, et al. Segregation of a missense mutation in the amyloid precursor protein gene with familial Alzheimer's disease. Nature. 1991;349(6311):704-706.

109. DeTure MA, Dickson DW. The neuropathological diagnosis of Alzheimer's disease. Mol Neurodegener. 2019;14(1):32.

110.Schliebs R. Basal forebrain cholinergic dysfunction in Alzheimer's disease - interrelationship with beta-amyloid, inflammation and neurotrophin signaling. Neurochem Res. 2005;30(6-7):895-908

111. Sang Q, Young HM. Chemical coding of neurons in the myenteric plexus and external muscle of the small and large intestine of the mouse. Cell Tissue Res. 1996;284(1):39-53.

112. Cabal A, et al. beta-Amyloid precursor protein (beta APP) in human gut with special reference to the enteric nervous system. Brain Res Bull. 1995;38(5):417-423.

113. Shankle WR, et al. Studies of the enteric nervous system in Alzheimer disease and other dementias of the elderly: enteric neurons in Alzheimer disease. Mod Pathol. 1993;6(1):10-14.

114. Borsom EM, et al. Do the bugs in your gut eat your memories? Relationship between gut microbiota and Alzheimer's disease. Brain Sci. 2020;10(11):E814.

115. Sohrabi M, et al. Gut inflammation induced by dextran sulfate sodium exacerbates amyloid- $\beta$ plaque deposition in the AppNL-G-F mouse model of Alzheimer's disease. J Alzheimers Dis. 2021;79(3):1235-1255.

116. Han X, et al. Loss of nitrergic and cholinergic neurons in the enteric nervous system of APP/ PS1 transgenic mouse model. Neurosci Lett. 2017;642:59-65.

117. Colombo AV, et al. Microbiota-derived short chain fatty acids modulate microglia and promote $A \beta$ plaque deposition. Elife. 2021;10:e59826.

118. Lin SY, et al. Dementia and vagotomy in Taiwan: a population-based cohort study. BMJ Open. 2018;8(3):e019582.

119. Sun $Y$, et al. Intra-gastrointestinal amyloid- $\beta 1-42$ oligomers perturb enteric function and induce
Alzheimer's disease pathology. J Physiol. 2020;598(19):4209-4223.

120. Lorenzi RM, et al. Unsuspected involvement of spinal cord in Alzheimer disease. Front Cell Neurosci. 2020;14:6.

121. Dugger BN, et al. The distribution of phosphorylated tau in spinal cords of Alzheimer's disease and non-demented individuals. JAlzheimers Dis. 2013;34(2):529-536.

122. Nopoulos PC. Huntington disease: a single-gene degenerative disorder of the striatum. Dialogues Clin Neurosci. 2016;18(1):91-98.

123. Walker FO. Huntington's disease. Lancet. 2007;369(9557):218-228.

124. Cowan CM, Raymond LA. Selective neuronal degeneration in Huntington's disease. Curr Top Dev Biol. 2006;75:25-71.

125. van der Burg JM, et al. Gastrointestinal dysfunction contributes to weight loss in Huntington's disease mice. Neurobiol Dis. 2011;44(1):1-8.

126. Moffitt $\mathrm{H}$, et al. Formation of polyglutamine inclusions in a wide range of non-CNS tissues in the HdhQ150 knock-in mouse model of Hunting ton's disease. PLoS One. 2009;4(11):e8025.

127. McCourt AC, et al. Characterization of gastric mucosa biopsies reveals alterations in Huntington's disease. PLoS Curr. 2015;7:ecurrents. hd.858b4cc7f235df068387e9c20c436a79.

128. Wood NI, et al. Increased thirst and drinking in Huntington's disease and the R6/2 mouse. Brain Res Bull. 2008;76(1-2):70-79.

129. Robberecht W, Philips T. The changing scene of amyotrophic lateral sclerosis. Nat Rev Neurosci. 2013;14(4):248-264.

130. Hardiman O, et al. Amyotrophic lateral sclerosis. Nat Rev Dis Primers. 2017;3:17071.

131. Herdewyn S, et al. Prevention of intestinal obstruction reveals progressive neurodegeneration in mutant TDP-43 (A315T) mice. Mol Neurodegener. 2014;9:24.

132. Anitha M, et al. GDNF rescues hyperglycemiainduced diabetic enteric neuropathy through activation of the PI3K/Akt pathway. JClin Invest. 2006;116(2):344-356

133. Luesma MJ, et al. New insights into c-Ret signalling pathway in the enteric nervous system and its relationship with ALS. Biomed Res Int. 2014;2014:328348.

134. McAlary L, et al. Prion-like propagation of protein misfolding and aggregation in amyotrophic lateral sclerosis. Front Mol Neurosci. 2019;12:262.

135. Scheperjans F, et al. Gut microbiota are related to Parkinson's disease and clinical phenotype. Mov Disord. 2015;30(3):350-358.

136. Alam MZ, et al. A possible link of gut microbiota alteration in type 2 diabetes and Alzheimer's disease pathogenicity: an update. CNS Neurol Disord Drug Targets. 2014;13(3):383-390.

137. Sampson TR, et al. Gut microbiota regulate motor deficits and neuroinflammation in a model of Parkinson's disease. Cell. 2016;167(6):1469-1480.

138. Lal S, et al. Vagal afferent responses to fatty acids of different chain length in the rat. Am JPhysiol Gastrointest Liver Physiol. 2001;281(4):G907-G915.

139. Huffman WJ, et al. Modulation of neuroinflammation and memory dysfunction using percutaneous vagus nerve stimulation in mice. Brain Stimul. 2019;12(1):19-29.
140.Erny D, et al. Host microbiota constantly control maturation and function of microglia in the CNS. Nat Neurosci. 2015;18(7):965-977.

141. Qiao CM, et al. Sodium butyrate exacerbates parkinson's disease by aggravating neuroinflammation and colonic inflammation in MPTP-induced mice model. Neurochem Res. 2020;45(9):2128-2142.

142. Hou Y, et al. Neuroprotective effects of shortchain fatty acids in MPTP induced mice model of Parkinson's disease. Exp Gerontol. 2021;150:111376.

143. Sampson TR, et al. A gut bacterial amyloid promotes $\alpha$-synuclein aggregation and motor impairment in mice. Elife. 2020;9:e53111.

144. Yarandi SS, et al. Intestinal bacteria maintain adult enteric nervous system and nitrergic neurons via toll-like receptor 2-induced neurogenesis in mice. Gastroenterology. 2020;159(1):200-213.

145. Mertsalmi TH, et al. Antibiotic exposure and risk of Parkinson's disease in Finland: a nationwide case-control study. Mov Disord. 2020;35(3):431-442.

146.Phillips RJ, et al. Alpha-synuclein-immunopositive myenteric neurons and vagal preganglionic terminals: autonomic pathway implicated in Parkinson's disease? Neuroscience. 2008;153(3):733-750.

147. Zha L, et al. Salmonella infection in chronic inflammation and gastrointestinal cancer. Diseases. 2019;7(1):E28.

148.Koscso B, et al. Gut-resident CX3CR1(hi) macrophages induce tertiary lymphoid structures and IgA response in situ. Sci Immunol. 2020;5(46):eaax0062.

149. Horuluoglu BH, et al. PAM3 protects against DSS-induced colitis by altering the M2:M1 ratio. Sci Rep. 2020;10(1):6078.

150. Kishimoto Y, et al. Chronic mild gut inflammation accelerates brain neuropathology and motor dysfunction in $\alpha$-synuclein mutant mice. Neuromolecular Med. 2019;21(3):239-249.

151. Norris EH, et al. Pesticide exposure exacerbates alpha-synucleinopathy in an A53T transgenic mouse model. Am J Pathol. 2007;170(2):658-666.

152. Olsen LK, et al. A role for viral infections in Parkinson's etiology? Neuronal Signal. 2018;2(2):NS20170166.

153. Wan QY, et al. Older patients with IBD might have higher risk of Parkinson's disease. Gut. 2020;69(1):193-194.

154. Cirstea MS, et al. Microbiota composition and metabolism are associated with gut function in Parkinson's disease. Mov Disord. 2020;35(7):1208-1217.

155. Dodiya HB, et al. Chronic stress-induced gut dysfunction exacerbates Parkinson's disease phenotype and pathology in a rotenone-induced mouse model of Parkinson's disease. Neurobiol Dis. 2020;135:104352.

156. Pelz JO, et al. Axonal degeneration of the vagus nerve in Parkinson's disease-a high-resolution ultrasound study. Front Neurol. 2018;9:951.

157. Choi JG, et al. High-throughput $16 \mathrm{~S}$ rRNA gene sequencing reveals that 6-hydroxydopamine affects gut microbial environment. PLoS One. 2019;14(8):e0217194.

158. Sun J, et al. Fecal microbiota transplantation alleviated Alzheimer's disease-like pathogenesis in APP/PS1 transgenic mice. Transl Psychiatry. 
2019;9(1):189.

159. Kim MS, et al. Transfer of a healthy microbiota reduces amyloid and tau pathology in an Alzheimer's disease animal model. Gut. 2020;69(2):283-294.

160.Raunio A, et al. Lewy-related pathology exhibits two anatomically and genetically distinct progression patterns: a population-based study of Finns aged 85. Acta Neuropathol. 2019;138(5):771-782.

161. Braak H, et al. New aspects of pathology in Parkinson's disease with concomitant incipient Alzheimer's disease. J Neural Transm Suppl. 1996;48:1-6.

162. Galloway PG, et al. Filaments of Lewy bodies contain insoluble cytoskeletal elements. Am J Pathol.1992;140(4):809-822.

163. Galvin JE, et al. Monoclonal antibodies to purified cortical Lewy bodies recognize the mid-size neurofilament subunit. Ann Neurol. 1997;42(4):595-603.

164. Walker DG, et al. Changes in properties of serine 129 phosphorylated $\alpha$-synuclein with progression of Lewy-type histopathology in human brains. Exp Neurol. 2013;240:190-204.

165.Forno LS. Pathology of Parkinson's disease. In: Marsden CD, Fahn S, eds. Movement Disorders. Butterworth-Heinemann; 1981:25-40.

166. Magrinelli F, et al. Pathophysiology of motor dysfunction in Parkinson's disease as the rationale for drug treatment and rehabilitation. Parkinsons Dis. 2016;2016:9832839.

167. Mazzoni P, et al. Motor control abnormalities in Parkinson's disease. Cold Spring Harb Perspect Med. 2012;2(6):a009282.

168. Lichter DG, et al. Cognitive and motor dysfunction in Parkinson's disease. Clinical, performance, and computed tomographic correlations. Arch Neurol. 1988;45(8):854-860.

169. Brown RG, Jahanshahi M. Cognitive-motor dysfunction in Parkinson's disease. Eur Neurol. 1996;36 (Suppl 1):24-31.

170.Poewe W, et al. Diagnosis and management of Parkinson's disease dementia. Int J Clin Pract. 2008;62(10):1581-1587.

171. Rickards H. Depression in neurological disorders: Parkinson's disease, multiple sclerosis, and stroke. J Neurol Neurosurg Psychiatry. 2005;76(Suppl 1):i48-i52.

172. Marsh L. Depression and Parkinson's disease: current knowledge. Curr Neurol Neurosci Rep. 2013;13(12):409.

173. Walsh K, Bennett G. Parkinson's disease and anxiety. Postgrad Med J. 2001;77(904):89-93.

174. Chen JJ, Marsh L. Anxiety in Parkinson's disease: identification and management. Ther Adv Neurol Disord. 2014;7(1):52-59.

175. Schrempf W, et al. Sleep disorders in Parkinson's disease. J Parkinsons Dis. 2014;4(2):211-221.

176. Lyons KE, Pahwa R. The impact and management of nonmotor symptoms of Parkinson's disease. Am JManag Care. 2011;17(Suppl 12):S308-S314.

177. Stacy M. Nonmotor symptoms in Parkinson's disease. Int J Neurosci. 2011;121(Suppl 2):9-17.

178. Cloud LJ, Greene JG. Gastrointestinal features of Parkinson's disease. Curr Neurol Neurosci Rep. 2011;11(4):379-384.
179. Chen QQ, et al. Gut inflammation in association with pathogenesis of Parkinson's disease. Front Mol Neurosci. 2019;12:218.

180. Houser MC, Tansey MG. The gut-brain axis: is intestinal inflammation a silent driver of Parkinson's disease pathogenesis? NPJ Parkinsons Dis. 2017;3:3.

181. Dutta SK, et al. Parkinson's disease: the emerging role of gut dysbiosis, antibiotics, probiotics, and fecal microbiota transplantation. J Neurogastroenterol Motil. 2019;25(3):363-376.

182. Postuma RB, et al. Identifying prodromal Parkinson's disease: pre-motor disorders in Parkinson's disease. Mov Disord. 2012;27(5):617-626.

183. Kikuchi A, et al. Hypometabolism in the supplementary and anterior cingulate cortices is related to dysphagia in Parkinson's disease: a crosssectional and 3-year longitudinal cohort study. BMJOpen. 2013;3(3):e002249.

184.Johnston BT, et al. Swallowing and esophageal function in Parkinson's disease. Am J Gastroenterol.1995;90(10):1741-1746.

185. Sheard JM, et al. Malnutrition in a sample of community-dwelling people with Parkinson's disease. PLoS One. 2013;8(1):e53290.

186.Forsyth CB, et al. Increased intestinal permeability correlates with sigmoid mucosa alpha-synuclein staining and endotoxin exposure markers in early Parkinson's disease. PLoS One. 2011;6(12):e28032.

187. Ramos Bernardes da Silva Filho S, et al. Neurodegeneration profile of Alzheimer's patients: a brain morphometry study. Neuroimage Clin. 2017;15:15-24.

188. Braak H, et al. Stages of the pathologic process in Alzheimer disease: age categories from 1 to 100 years. J Neuropathol Exp Neurol. 2011;70(11):960-969.

189. Tiraboschi P, et al. Alzheimer disease without neocortical neurofibrillary tangles: "a second look". Neurology. 2004;62(7):1141-1147.

190.Albers MW, et al. At the interface of sensory and motor dysfunctions and Alzheimer's disease. Alzheimers Dement. 2015;11(1):70-98.

191. Buchman AS, Bennett DA. Loss of motor function in preclinical Alzheimer's disease. Expert Rev Neurother. 2011;11(5):665-676.

192. Wirths O, Bayer TA. Motor impairment in Alzheimer's disease and transgenic Alzheimer's disease mouse models. Genes Brain Behav. 2008;7(Suppl 1):1-5.

193. Aggarwal NT, et al. Motor dysfunction in mild cognitive impairment and the risk of incident Alzheimer disease. Arch Neurol. 2006;63(12):1763-1769.

194. Wilson RS, et al. The natural history of cognitive decline in Alzheimer's disease. Psychol Aging. 2012;27(4):1008-1017.

195. Kelley BJ, Petersen RC. Alzheimer's disease and mild cognitive impairment. Neurol Clin. 2007;25(3):577-609.

196. Ownby RL, et al. Depression and risk for Alzheimer disease: systematic review, meta-analysis, and metaregression analysis. Arch Gen Psychiatry. 2006;63(5):530-538.

197. Kaiser NC, et al. Differences in anxiety among patients with early-versus late-onset Alzhei- mer's disease. J Neuropsychiatry Clin Neurosci. 2014;26(1):73-80.

198.Ferretti L, et al. Anxiety and Alzheimer's disease. J Geriatr Psychiatry Neurol. 2001;14(1):52-58.

199.Teri L, et al. Anxiety of Alzheimer's disease: prevalence, and comorbidity. J Gerontol A Biol Sci Med Sci. 1999;54(7):M348-M352.

200.Brzecka A, et al. Sleep disorders associated with Alzheimer's disease: a perspective. Front Neurosci. 2018;12:330.

201. Bliwise DL. Sleep disorders in Alzheimer's disease and other dementias. Clin Cornerstone. 2004;6(Suppl 1A):S16-S28.

202.Kohler CA, et al. The gut-brain axis, including the microbiome, leaky gut and bacterial translocation: mechanisms and pathophysiological role in Alzheimer's disease. Curr Pharm Des. 2016;22(40):6152-6166.

203.Secil Y, et al. Dysphagia in Alzheimer's disease. Neurophysiol Clin. 2016;46(3):171-178.

204.Kowalski K, Mulak A. Brain-gut-microbiota axis in Alzheimer's disease. J Neurogastroenterol Motil. 2019;25(1):48-60.

205.Jiang C, et al. The gut microbiota and Alzheimer's disease. J Alzheimers Dis. 2017;58(1):1-15.

206.Ikeda M, et al. Changes in appetite, food preference, and eating habits in frontotemporal dementia and Alzheimer's disease. J Neurol Neurosurg Psychiatry. 2002;73(4):371-376.

207.Fu P, et al. Association of intestinal disorders with Parkinson's disease and Alzheimer's disease: a systematic review and meta-analysis. ACS Chem Neurosci. 2020;11(3):395-405.

208.Malek N, Newman EJ. Hereditary chorea - what else to consider when the Huntington's disease genetics test is negative? Acta Neurol Scand. 2017;135(1):25-33.

209.Smith MA, et al. Motor disorder in Huntington's disease begins as a dysfunction in error feedback control. Nature. 2000;403(6769):544-549.

210. Peavy GM, et al. Cognitive and functional decline in Huntington's disease: dementia criteria revisited. Mov Disord. 2010;25(9):1163-1169.

211. Epping EA, Paulsen JS. Depression in the early stages of Huntington disease. Neurodegener Dis Manag. 2011;1(5):407-414.

212.Dale M, van Duijn E. Anxiety in Huntington's disease. J Neuropsychiatry Clin Neurosci. 2015;27(4):262-271.

213. Herzog-Krzywoszanska R, Krzywoszanski L. Sleep disorders in Huntington's disease. Front Psychiatry. 2019;10:221.

214.Arnulf I, et al. Rapid eye movement sleep disturbances in Huntington disease. Arch Neurol. 2008;65(4):482-488.

215. Stan TL, et al. Increased intestinal permeability and gut dysbiosis in the R6/2 mouse model of Huntington's disease. Sci Rep. 2020;10(1):18270.

216.Kagel MC, Leopold NA. Dysphagia in Huntington's disease: a 16-year retrospective. Dysphagia. 1992;7(2):106-114.

217. Toepfer M, et al. Delayed colonic transit times in amyotrophic lateral sclerosis assessed with radioopaque markers. EurJMed Res. 1997;2(11):473-476.

218.Zeng $\mathrm{Q}$, et al. The alteration of gut microbiome and metabolism in amyotrophic lateral sclerosis patients. Sci Rep. 2020;10(1):12998. 